\title{
Biological Stress as a Principle of Nature: A Review of Literature
}

\author{
Celia Martins Cortez ${ }^{1}$, Dilson Silva ${ }^{2}$ \\ ${ }^{1}$ Department of Applied Mathematics, Rio de Janeiro State University, UERJ, Rio de Janeiro, Brazil \\ ${ }^{2}$ Biomanguinhos, Foundation Oswaldo Cruz, FIOCruz, Rio de Janeiro, Brazil \\ Email: ccortezs@ime.uerj.br
}

How to cite this paper: Cortez, C.M. and Silva, D. (2020) Biological Stress as a Principle of Nature: A Review of Literature. Open Journal of Biophysics, 10, 150-173. https://doi.org/10.4236/ojbiphy.2020.103012

Received: June 1, 2020

Accepted: July 19, 2020

Published: July 22, 2020

Copyright $\odot 2020$ by author(s) and Scientific Research Publishing Inc. This work is licensed under the Creative Commons Attribution International License (CC BY 4.0).

http://creativecommons.org/licenses/by/4.0/

\begin{abstract}
This review paper attempts to approximate the concept of biological stress to the stress concept in Physics using the phenomenological view of physics to discuss the source of generator forces of biological stress state. Based on the literature, parallels are drawn between the two concepts and a discussion on the steady state in open systems and homeostatic state in biological systems is developed. Using the concepts of thermodynamic entropy and informational entropy, and comparing stress in living systems and nonliving, we attempt to build a basis for a view of stress as a principle of nature linked to the adaptability property of matter, opposing entropy. It is known that the increasing number of microstates possible in a complex system increases the entropy. In that way, entropy is related to the amount of additional information needed to specify the exact physical state of a system, given its macroscopic specification. By controlling the metabolic processes (catabolism-anabolism) to decrease the entropy, stress reduces the number of possible states for which the living system could evolve, avoiding the loss of "life information", preserving its characteristics and preventing its extinction. The loss of function of a species within an ecosystem or of cells within an organ can be showing that the limits of the stress principle were "transgressed". That is, the intensity and/or duration of stress exceeded the capacity of living organism to process of information extracted from stressor and reprogram its physiological mechanisms, activating its adaptability process, while its internal balance is preserved.
\end{abstract}

\section{Keywords}

Stress Concept, Biological Stress, Thermodynamic Entropy, Information Entropy, State of Stress 


\section{Introduction}

For many people, Physics and Biology are different worlds, because each one has its own language and concepts. However, in recent decades, scientific and technological advances have created overlaps between these sciences, starting the life modeling age. According Chao et al. [1], you can open the door to the creation of virtual human reality, developing the ability to associate physiology and engineering knowledge with computer science.

Indeed, details of human physiology inaccessible to experimental studies have been enlightened by means of computational simulations using physicalmathematical models of physiological systems [2]-[16]. As a starting point, the modeling and simulation in Biology require analysis and representations of its phenomena from different points of view.

Physics knowledge has been applied to study mechanical and hemodynamic of effects of stress on the musculoskeletal and circulatory systems [17]-[22]. Computer simulations have been used to verify the distribution of stress forces in blood vessel to find the structural determinants for atheromatous plaque vulnerability [23] [24]. The evolution of these plaque has been studied using growth kinetic models based on oxidized LDL (low-density lipoprotein) accumulation and mass conservation concept [25] [26], and including the endothelial permeability dependence of stress forces and other parameters [27]. Physical and mathematical modeling and computational simulation have also been applied to investigate the mechanisms underlying neurovascular coupling involving nitric oxide [28] [29].

In addition, forces involved in various types of cell motility have been modeled. The mobility of cytoskeletal filaments (stretching fast polymerization/ depolymerization) and the influence of their length on the mechanical response have been investigated, using cellular force-generation models. The thermodynamic driving force for force-generation in these processes has been investigated, and the elastic-Brownian ratchet model for force generation seems valid to study the monomer addition to free cytoskeletal filament ends [30] [31] [32].

Despite the several studies and the physical-biological modeling in the description of phenomena involving stress forces acting in physiological and pathological processes [33] [34], biologic stress continues to be the subject of great controversy, from its definition to its pathophysiological inferences. Sometimes the word stress is used to denote a complex response developed by a stimulus. However, at other times, stress is mistakenly assumed to be a stimulus capable of causing illness or is used as a synonym for "illness". In addition, alarm reactions are often called stress, despite the existence of well-established criteria to differentiate them. It is also not uncommon to hear someone confusing anxiety with stress, forgetting that in the state of stress a set of specific systemic response occurs evoked by exposure to the stressor stimulus. This full set is not present in anxiety, but usually anxiety is present in stress states [35] [36] [37] [38]. 
The aim of this review is to approximate the concept of biological stress to the stress concept in Physics, not through mathematical modeling, but using the phenomenological view of physics to discuss the source of generator force of biological stress state. Based on the literature, parallels are drawn between the two concepts, and a discussion on entropy, steady state in open systems and homeostatic state in biological systems is developed.

\section{Concepts and Definitions of Stress}

In strict sense, the word stress means "pressure" or "tension" and be stressed means "be under pressure" or "be under the action of an insistent stimulus" [38] [39]. The concept of stress was introduced in the theory of elasticity by theoretical physicist Augustin-Louis Cauchy in the second decade of the $19^{\text {th }}$ century, based on conception of material continuity. According to the Cauchy's stress principle, when a continuous body is submitted to the action of external forces, internal reactions arise throughout the body, acting between material points [40]. Thus, in Physics, the word stress refers to a measure of the intensity of the total internal force acting within a body across imaginary internal surfaces [40] [41]. Therefore, stress means the "reaction" of the body to the action of external forces, which represent the stressor [38] [39] [42].

In biology, the stressor is any stimulus or event capable of establishing the state of stress. Stress is a reactive process to reduce the negative effects caused by the stressor, and stress of state is a state of resistance that aims to maintain the internal equilibrium of the body (and mind), whose final purpose is to adapt the individual to the stressor [39] [43] [44].

Therefore, in Biology, the term stress also means "reaction". It is not an instantaneous and localized reaction, but a reactive process that triggers a typical set of organic and behavioral responses, which has as central pattern the hyperfunction of the supra-renal gland and the increased release of glucocorticoids (GC), especially cortisol in humans [45].

\subsection{Stress in Material Body}

The stress $(\sigma)$ is the measurement of the average intensity of all forces acting per area unit of a body submitted to a stressor stimulus, and can be represented by the equation:

$$
\sigma=\frac{F}{A}
$$

where $F$ is the force acting over area $A$, and the unit of pressure in the SI is normally used for stress. In that way, stress has the meaning of pressure on the surface $A$, and Equation (1) is valid when the action of stressor is considered uniformly distributed over a transversal section of the material body. However, in general, it is not truth, and consequently stress at a point on a given area is different from the average stress over the entire cross section. In that case, stress can be defined at a specific point in body [40] [46]. 
Cauchy demonstrated that a second order cartesian tensor (Cauchy's stress tensor) was able to completely define the state of stress at any point in a body. A tensor is a form of representation associated with a set of algebraic operations such as the sum and the product, related to a vector space. The linear stress $\left(\sigma_{i j}\right)$, which represents unidimensional stress, is a tensorial entity and can be represented by a $3 \times 3$ matrix. Using this matrix, it is possible to formulate that the force vector (or traction vector) acting on an surface element is given by

$$
F=\sum_{j}^{3} \sigma_{i j} n_{j}
$$

where $n_{j}$ denotes the unit vector normal to the surface element. The Cauchy's stress tensor $\left(\sigma_{i j}\right)$ is used for the stress analysis of material bodies experiencing small deformations, being other measures of stress used for large deformations [40].

Here, it is important to pay attention to fact that the deformations result from the "struggle waged" between stressor and stress, and represent the victory of the stress forces over forces contrary to the cohesion responsible for the "existence" of the material body. Deformations are inherent changes in the adaptation process, which is established to preserve the body components that, even in a new configuration, still remain in cohesion.

In Physics, stress cannot be directly measured, but is usually estimated from strain measurements and elastic properties of the material, since the stress tensor is associated with the strain required for the body adapts to the stressor and keep its internal equilibrium. In equilibrium, the components of the stress tensor at each point of the body satisfy the equilibrium equations, i.e., $\sigma_{i j, i}+F_{i}=0$. In addition, in equilibrium, the summation of moments on an arbitrary point is zero, indicating that the tensor is symmetric $\left(\sigma_{i j}=\sigma_{i j}\right)$ [40] [46].

\subsection{Definitions of Stress in Living Systems}

All organs and living tissues have their own mechanisms to keep its internal balance, i.e., our body has the homeostatic property. Homeostasis is an ability of the open systems (that interact directly with its environment), especially living organisms, to regulate its internal environment in order to maintain a stable state. This is achieved by means of multiple mechanisms appropriate to promote dynamic equilibrium adjustments.

In the animal body, the dynamic equilibrium or dynamic homeostasis is characterized by a constant regulation of biophysical variables (blood pressure, temperature, salinity, acidity), nutrient concentrations (glucose, various ions, oxygen) and waste, as carbon dioxide, urea etc. [47] [48].

According to Jackson [49], ten characteristics define an open system, i.e., all open systems: 1) import energy from the external environment; 2) process and transform everything they receive from the environment; 3) export what they produce to the environment; 4) serve as new sources of energy; 5) have negative entropy, since they obtain more energy than they spend; 6) selectively collect in- 
formation about their environment and their activities, and are corrected based on this information; 7) maintain their character due to the dynamic homeostasis; 8) are capable of differentiate and develop their structures; 9) have integrate functioning; 10) obtain the same effect from different events (equifinality).

Claude Bernard (1813-1878) was who proposed the concept of stability in biological systems, which was called homeostasis by Walter Cannon [50], in 1914. Canon's and Bernard's studies showed the role of adrenal epinephrine (or adrenaline) in the homeostatic process, while Hans Selye [45] demonstrated, in 1946, the adaptive importance of adrenal GC, analyzing the wide scope of action of these hormones in the body. Precisely, homeostasis is a property of all living systems, and several homeostatic mechanisms are active full-time to keep internal balance.

Selye [45] called the sum of all the systemic non-specific reactions of the body resulting from a long-continued exposure to stressor of Syndrome of General Adaptation. It is distinct from specific adaptive reactions, such as the development of the musculature following prolonged physical exercise and the immunologic and allergic phenomena. Syndrome of General Adaptation is divided in three different stages: 1) stage of alarm reaction, in which the subject experiences characteristic sensations from alterations in the internal equilibrium of the body; 2) stage of resistance, when the organism tries to adapt to the stressor, and 3) stage of exhaustion, which results from prolonged exposure to the stressor without occurrence of adaptation. Psychosomatic diseases are a result of the organic inability to compensate for stress. Selye emphasized the implication of the sustained high production of GC to the establishing of the exhaustion stage.

Thus, stressor stimulus initially provokes the state of alarm, due to the strong sympathetic discharge and consequent release of catecholamines, norepinephrine and epinephrine, from the adrenal medulla, which produce characteristic cardiovascular and metabolic effects. If the action of the stressor is of short duration or not intense, sympathetic and catecholaminergic effects can be able to maintain the internal equilibrium of the body. However, sustained exposure to the stressor action provokes the increased release of GC [45], establishing the state of resistance or stress. This prepares the organism to satisfy the energetic demand and to increase the general defense mechanism.

The state of stress is established when a wide, intense and prolonged reaction is necessary to maintain this internal balance. The stress hormone (cortisol, in human) is able to directly and indirectly initiate and control all stress mechanisms, seeking to maintain of internal equilibrium and adapt the animal to the action of the stressor or stressing situation. The stressor stimulates the state of stress and the forces generated by physiological responses (hormonal, neuromuscular, cardiovascular, inflammatory and immune) are resistance forces [39] [45] [48].

Almost every major life change can be considered as a stressors, i.e., changes that require non-ordinary increase in metabolic dynamics to keep the organic balance and/or emotional. Thus, the stress state is characterized by energy ex- 
penditure greater than that the body/mind is habituated in its daily life [51].

Effects of the Stress Hormone. GC have positive and negative effects in the dependence of their plasmatic level and time of exposure to stressor. In normal conditions, GC regulate with considerable degree of specificity the cellular differentiation in several tissues. GC can accelerate or delay the functional organic maturation. Normally, these hormones help in the general body growth and formation and maturation of organs, functioning together with GH (growth hormone), thyroid hormones, adrenal androgens and sexual hormones [52] [53]. However, GC excess can exert negative effects on body growth, inhibiting spontaneous secretion of the cited hormones and influencing negatively on cell proliferation [10] [54]-[59].

The most pronounced GC effect is its capacity to increase the blood glucose from hepatic gluconeogenesis, but it also plays a role in cardiovascular, nervous, endocrine, inflammatory and immunological responses [48] [60]. Very increased cortisolaemia causes: 1) the increase in plasmatic concentrations of glucose, amino acid and free fatty acid from cellular reserves; 2) decreased synthesis and increased catabolism of certain proteins; 3) alterations in hydroelectrolitic balance, circulatory hemodynamics and autonomic responses; and 4) decrease in inflammatory and immunological responses [61] [62] [63].

CG influence the phenotypical expression at synapses. It was observed that they increase the noradrenergic responses in cell populations predominantly cholinergic in its absence, evidencing its potential to change synaptic properties and to regulate neuronal plasticity [53] [64]. These observations are very important, considering that synapses are the base of neuronal processing.

On the inflammatory responses and immunological, GC play an important regulatory roles; They inhibit enzymes involved in the production of inflammatory substances, reduce the number of circulating lymphocyte (lymphopenia), eosinophil and neutrophil, and inhibit the antibody production [64] [65] [66] [67] [68]. High GC-exposure can produce significant atrophy on lymphoid tissues and contribute to the pathogeny of inflammatory diseases and to clinical progression in viral infections [67] [69] [70] [71] [72].

Indeed, mechanisms developed during the stress states may become pathogenic, depending on the following factors: 1) The stress intensity; 2) stress duration, since protein synthesis does not decrease during the first 24 hours after the increase of GC, and an immediate increase in DNA synthesis can be observed; 3) affective representation of the stressor; 4) nutritional status of the individual; 5) extension periods of rest and sleep [38] [55] [58].

Efforts have been made in the search for objective markers to assess the intensity of stress and its effects. In experiments with rats, stress has been measuring by blood levels of GC, especially corticosterone [54] [73] [74]. In humans, cortisol is a natural marker, which can be measured in the blood, saliva and hair [75] [76] [77].

In conjunction with cortisol levels, other biological measurements (such as salivary prolactin, vasopressin, blood pressure and heart rate variability) have been 
considered useful to monitor the evolution of stress and validate stress reduction programs [78] [79] [80]. But it is important consider racial/ethnic and social class differences to study association between stress level and cortisol secretion. Physiological measurements may be convenient to indicate the stress in animals, however, the study of the state of stress in humans requires more detailed analysis [81] [82] [83].

\section{Biological Systems and Internal Balance}

Understanding the phenomenon of biological stress from the point of view of Physics requires analysis of the stress state in its specific aspects looking for conceptual convergences.

Based on the concept of stress and the physiological mechanisms involved and implications, we could say that a stressed organism is in "excited state". This is a term commonly used in the context of atomic/molecular phenomenology [84]. In fact, during stress, the body is out of its "ground state", and it is in a "higher energy level" or "excited state". As expected for an excited molecule, the natural tendency of "excited organism" would be after some time go to back to its "lower energy state". However, here are worth the following questions: what would a biological ground state or lower energy state be? Does it make sense to talk about a ground state in Biology?

\subsection{Ordinary and Stress Homeostatic State}

In the search for answers to the questions above, we will start considering that each cell has its condition of maximum balance, that is, a condition in which its components work in their "ideal operating conditions", thus spending the least amount of energy possible to maintain its functions. Undoubtedly, maintaining an "ideal" work rate in a cell requires certain conditions, such as the absence of morphological and metabolic errors. In addition, the environment must be able to meet all its chemical, physical and nutritional needs. That way, we could have a cell operating in "fundamental condition" ... Would that be possible?

But, these conditions are nearly impossible to meet. However, we can assume that each cell (or each part of the body) can have its own "lower energy state" in accordance with its morphofunctional conditions. This would be its state of higher internal possible balance, its "steady state" or homeostatic state. In this, the physiological parameters can vary within certain limits, being adjusted by immediate response mechanisms, which do not mobilize large energy resources. Variations out homeostatic limits call for intervention of more powerful and wide control mechanisms, which are not active in the ordinary steady state. Such mechanisms involve the mobilization of energetic reserves do not used in homeostatic conditions, such as gluconeogenesis and proteolysis to generate energy. At that moment, the state of stress or "excited" is established.

Despite the large increase in the energy consumption during the state of stress, such as occurs in the ordinary homeostatic state, energy enters and leaves the system at the same speed, and physiological parameters remain within de- 
fined limits, due to the continuous production of energy and matter [49] [85]. However, it is important to consider that, extinguished the action of stressor, it is possible that living organism does not return to its homeostatic state before stress. In post-stress, depending on how long and/or severe the stress was, functional balance may be moved to another level, in which organic activity and energetic expenditure can be higher or smaller from the previous one.

So, we can say that the cell/organism is in an excited state during the stress and, and it may or may not return to the work regime prior to the stress period with the suspension or exhaustion of the resistance forces.

After prolonged stress, there is great chance of the organism starts operating in a regime of lower metabolic activity, with changes in the rate of transfer and transformation of energy/matter, and physiological deficits due to the depletion of mobilized resources [51]. Post-stress alterations can result in major imbalances and result in deep functional changes in tissues and organs [39] [54] [73] [74] [86], including endocrine, metabolic and immunological changes. There are studies shown that cytokines play an important role in these changes [87] [88] [89].

\subsection{Internal Imbalance and Illness}

The function of stress is to keep the biological system in balance, controlling the production and expenditure of energy and matter, conserving the catabolism-anabolism balance and taking into account energetic, structural and transport demands [90].

One of the important consequences of catabolism-anabolism disequilibrium is the cellular oxidative stress. This results from excessive formation of free radicals (substances with high oxidation capacity) due to an irregular balance between the formation rate of these radicals and the neutralizing capacity of arsenal of antioxidant enzymes. The mechanisms of oxidative stress interfere in cellular functions.

Much emphasis has been placed on nuclear and membrane changes produced by lipid peroxidation and cell protein degradation induced by oxidative stress. Due to their aggressive potential, free radicals have been identified as carcinogenic and atherogenic substances, and capable to induce neurodegenerative diseases (such as Alzheimer's and Parkinson's diseases) and aging [91]-[96].

In 2004, Epel et al. [96] studied the relationship between telomerase activity and telomere length in mononuclear blood cells from healthy women. Telomeres are repeated sequences of DNA that form the end (tip) of all linear chromosomes. Telomerase is a ribonucleoprotein enzyme (is a reverse transcriptase) that stabilizes the telomere length. This enzyme adds hexametric repeats to the ends of telomeres of the chromosomes, compensating the continued telomere erosion [97] [98]. Epel et al. [99], in 2006, observed lower telomerase activity and shorter telomeres in women with higher levels of perceived stress. The average telomere shortening was equivalent to at least a decade of additional aging compared with low stress women. 
According Kiecolt-Glaser and Glaser [100], the studies of Epel and colleagues allowed to understand the link among chronic psychological stress, oxidative stress and telomere length since psychological stress can increase inflammation and oxidative stress [101]. It has been shown that the telomere attrition is accelerated by both inflammation and oxidative stress [102] [103]. There are several recent evidences indicating that telomeres and telomerase activity are particularly sensitive to oxidative damage and psychological stress [104] [105] [106].

More recently, other studies have discussed the impact of stress, especially psychological stress, on the aging process [107] [108] [109]. The important role that stress plays in senescence process has been demonstrated. In this process there is a gradual decrease in cellular functions and a consequent reduction in cell multiplication. It is a process linked to aging, in which the shortening of telomeres is a key mechanism [110] [111].

Increased rates of morbidity and mortality from chronic diseases of aging are found in individuals chronically exposed to psychological stressors early in life [112]. Some diseases related to aging have been linked to the action of stressors, and epigenetic alterations have been identified as an important mechanism that links stress to aging. In such alterations, environmental factors induce changes in gene expression, but the genetic sequence is preserved [107].

\section{Entropy and Biological Systems}

At steady state, the properties of any material body can be described by thermodynamics; and analysis of biological stress in this way can reveal other distinctive features of its nature.

The second law of thermodynamics is related directly to a concept widely discussed in Biology: entropy $(S)$ [113]. In 1865, Rudolf Julius Emanuel Clausius (1822-1888) introduced in Physics the concept of entropy, from the Greek en-tropein, which means "the transformative content" or "the content of transformation" [114].

Just like stress, entropy is a complex subject, and different ways have been used to understand its concept.

Entropy is an abstract dimension that represents the measure of disorder of particles in a physical system, as well as the measure of randomness and irreversible increase in energy in the universe. It is an entity involved in changes that occur in our universe constantly moving and transformation, being therefore difficult to represent it in a totally clear form [46] [115] [116].

According to the second law of thermodynamics, "the entropy of an isolated system not in thermodynamic equilibrium will tend to increase over time, approaching a maximum value at the equilibrium", i.e., the system reaches a state of maximum entropy.

As a function of the state, the change in the entropy of a system is determined by its initial and final states, being constant in reversible processes and, in irreversible processes, the total entropy continues to increase. That is, in a system in equilibrium entropy is high, and no spontaneous process can occur without in- 
creasing the total entropy of the universe ( $S>0$, for spontaneous processes). In living systems, as all highly organized systems, entropy is very high. [115]

In macroscopic thermodynamics, the concept of entropy is related to the difference between the useful chemical energy and dissipated energy. Transferring an amount of heat to a system in equilibrium, the increase in entropy can be represented by $\delta Q / T$, where $\delta Q$ is the energy flow into the system due to heating and $T$ is the absolute temperature. The total entropy will be given by the integration: $\Delta S=\int \delta Q / T$, as defined by Rudolf Clausius [114]. The heating of system increases randomness of molecular motion and generating more microstates possible.

In complex systems, entropy is determined by the number of random microstates. The greater the number of possible microstates, the higher is the entropy [115] [117].

In statistical thermodynamics, the entropy is the measure of the degree of probability that a system will expand into different possible quantum states. In a system in equilibrium, entropy is maximized due to the loss of all information on the initial conditions, except for the stored variables. In view based on the probability Boltzmann (1872), the entropy of a macroscopic state is proportional to the logarithm of the number of microscopic states (states or density), is given by the equation

$$
S=-k \sum_{i} p_{i} \ln \left(p_{i}\right)
$$

where $k$ is the universal constant of proportionality and $p_{i}$ defines the individual probability for each microstate. Therefore, the statistical view shows entropy as the amount of uncertainty that remains in the system after considering its observable macroscopic properties. It leads to the idea that all dynamically ordered states are highly unlikely states, including life. Therefore, this view of the second law has been intensely debated [118].

The mathematical concept of thermodynamic entropy has been extended and interpreted in the light of Statistical Mechanics and Information Theory. These two entropy concepts present visible connections, and they play an important role in our discussion involving entropy and biological stress.

\subsection{Entropy in Statistical Mechanics and Living Systems}

Ludwig Eduard Boltzmann (1844-1906) tried to explain the existence of a "high degree of organization" in the universe through an argument called the paradox of the brains of Boltzmann. According to this, the low entropy system is a random fluctuation in a higher entropy universe. Even in a quasi-equilibrium state, stochastic fluctuations are present in the degree of entropy. But there was much opposition to the arguments of Boltzmann [119] [120].

According to Erwin Schrödinger (1887-1961), there is no violation of the equilibrium equation of the second law, if we consider that living beings produce entropy at a rate sufficient to compensate for their own internal ordering, concentrating a stream of order on itself, because "life feeds on negative entropy" 


\section{[121] [122].}

The problem of high improbability of spontaneous order started to be solved when Karl Ludwig von Bertalanffy (1952) showed that that open systems can order itself by their ability to build their order by dissipating potentials in their environments [123]. In 1977, Ilya Prigogine [124] called such systems "dissipative structures or self-organized" and contributed to the drafting of minimum production of the Theorem of Entropy, applicable to stationary states of non-equilibrium" [125]. This theorem gives a clear explanation of the analogy between the stability of thermodynamic equilibrium and stability in biological systems, generated by dynamic equilibrium, as expressed in the concept of homeostasis proposed by Claude Bernard. Thus, a living system self-organizes and continues self-organizing throughout its life, due to its metabolic dynamics [126] [127].

The concept of entropy was later extended by J.P. Lowe [127], based on the relevance of occupancy of energy levels, and H.S. Leff [128] provided a theoretical basis for interpreting the increase in entropy with the increase in energy dispersion due to rise of the number of microstates. In this case, entropy is associated with increased randomicity due to interparticle spacing. For an ideal gas expansion in vacuum, for example, the molecules can take different forms (electronic, vibrational, rotational and translational) to distribute their energy, generating different entropies.

\subsection{Information Entropy and Living Systems}

In Shannon's Information Theory (1948) the word entropy was included based in its concept in Statistical Thermodynamics, developing thus the information entropy. For Shannon, the number of distinguishable symbols in a communication channel is the number of distinguishable states in a system [129] [130].

The information entropy measures the uncertainty on the amount of information needed to represent a system. According Brillouin [131], acquiring information about the possible microstate (possible combinations of particles in possible energy states) of a system is associated with the decrease in entropy, since work is needed to extract information, and information removal leads to an increase in thermodynamic entropy. In that way, entropy is related to the amount of additional information needed to specify the exact physical state of a system, given its macroscopic specification. So, entropy can be also considered an expression of the lack of information about this state [132].

Information Entropy has also been used to display electronic properties as information functions for atoms and molecules. For Zhou et al. [133], Shannon entropy should contain all the information needed to adequately describe an electronic system.

The Functional Information Theory based on Shannon Theory has gained application in a wide range of studies in Biology and Medicine, including detection of gene-gene interactions, classification of biological compounds, and analysis of electrogram entropy maps in cardiology [134]-[143]. 
Using information theory, Ofria et al. [144] focuses on the selective pressures of molecular evolution and how they contribute to the development of robust and complex structures. Despite the information theory does not tell about information related to functions and mechanisms involved in cellular input-output, Lan and $\mathrm{Tu}$ [145] believe this theory provides a general tool able to analyze certain biological data.

In addition, this theory has suggested that genetic instability of repeated DNA sequences may be fundamentally related to the process of aging. According to Riggs [139], rather aging and mortality are the inevitable natural consequence of increasing informational entropy (decreasing redundancy) contained within the genome. Therefore, the information theory has progressing, and fundamental aspects of average entropy and mutual entropy mean in the open system dynamics has been presented [140] [141].

Thus, the view of informational entropy complements the thermodynamic view. In the first, entropy relates to the uncertainty of the information distribution and, the second refers about the loss of all information on the initial conditions in a system in equilibrium and entropy maximized.

\section{Stress as a Principle of Nature}

The present discussion aims to highlight the opposite roles of stress and entropy. While in the concept of entropy "life as highly unlikely", corresponding to an amount of uncertainty on the existence or measure of the degree of probability of a system expands into possible different microstates, the stress becomes the life highly probable, opposing to the expansion of system and preserving the number of possible state and its existence.

We draw attention to the fact that, although manifesting in different forms, stress is always the same basic and intrinsic principle of living or not living systems. The preservation of life involves forces ranging from molecular to the mental level, which generate conservative/adaptive behaviors. Thus, we highlight a possible way to understand stress as a principle of nature, considering the biological stress forces as a consequence of this principle wherein "the order is produced in order to bring back to order in order to produce more order".

In humans, the corporal and mental stress is the macroscopic manifestation or the translation of cellular stress, which develops from stress at the molecular level [146]. Thus, the biological stress is the result of the combination of reactive forces developing on an increasing scale, from the microscopic to macroscopic scale, face to the possibility of internal balance disruption of the living system: tissues, cells, biomolecules. Stress forces trigger specific responses to sustain the dynamic homeostasis and preserve the order, which defines the "information contained in the life".

From the thermodynamic point of view, the action of the stressor in a system tends to alter its entropy, since it changes its equilibrium and increases the randomicity, due to the increased number of possible states. In that way, stress mechanisms try reduce the uncertainty about the initial state of the system, as well 
as about the amount and distribution of information (genetic, and/or morphophysiological information, in biological systems) needed to specify its exact physical state. Maximizing entropy leads to maximum condition of ignorance about this state. But the forces of stress, opposing the stressor, seek to balance the entropic forces and protect the information responsible to maintaining the ordered system.

Like entropy, stress is not uniformly distributed throughout the living body. Each cell or tissue exhibits a specific response to stressor [38], and the control of catabolism-anabolism balance seems to be a way of dissipating internal entropy [90] [126] [127].

In our dual universe, where randomness coexists with ordering, biological stress is opposed to randomness to keep the in dynamic equilibrium until adaptation is achieved. However, when stress exceeds adaptive limit, stressful or entropic forces overcome the forces of stress and the organic imbalance grows. Then, the exhaustion stage is installed [45], generating physiological disorder that can progress to disease.

As some works in the literature show, regardless of the stressor, biological stress can be studied in the light of Physics. Models of biological stress can be built based on internal changes (metabolic, ionic gradients, membrane repair, among others) generated by resistance forces and the amount of energy spent on these changes. Predictive models can be built, and computer simulations can provide information about the average responsiveness of the living system to a specific stressor [18] [19] [20] [21] [22] [30] [31] [32] [147] [148] [149] [150] [151].

Due immense diversity of living systems, the application of mathematical formalism to study stress in these systems seems impossible. But, the work of Epel et al. [96], cited above, showed that, like the non-living systems studied in Physics, intense and/or prolonged stress can "deform" structures in the biological system, as the shortening that they observed in telomeres.

Although it is a difficult task, the current knowledge on organic and cellular structures, interactions and mechanisms triggered by stress already allows drawing parallels aiming to model the biological stress based on Physics concepts.

\section{Conclusions}

By means of the review and comments above, we attempt to present an alternative view of the biological stress using concepts of Physics. In the state of stress, conservative forces are activated trying to preserve the order of systems, enabling their existence.

Considering the concepts of thermodynamic entropy and informational entropy, and comparing stress in living and non-living systems, we can see it as a principle of nature linked to the adaptability property of matter, as opposed to entropy. It is known that increasing the number of possible microstates in a complex system increases entropy, since it increases the uncertainty about the 
additional information needed to specify the exact physical state of a system. By controlling metabolic processes (catabolism-anabolism) to decrease entropy, stress reduces the number of possible states into which the living system could evolve, preventing the loss of "life information", preserving its characteristics and preventing its extinction.

The animal organism has several efficient mechanisms to maintain its general balance during stressful situations. This mechanism, however, can fail since the cellular responses depend on the type and intensity of stress.

The cell can cope with effects from the stressor, if it does not exceed its capacity to generate adequate protective responses to ensure its survival. Thus, as seen above, very prolonged stress has a destructive effect on tissues, because excess organic GC inhibits several activities, including hormone secretion, cell proliferation, immune responses and causes significant nervous changes.

It is important to remember that any "machine", living or not, has its adaptation limit, outlined by its constitution and structure, and its mechanisms of interaction with the environment. The final changes, which result in an effective adaptation, must be processed within the speed limits compatible with the responsiveness of the "machine". The compatibility of these speeds allows the relaxation of machine components, between one and another processing. Relaxation time is the period of time required for a system out of equilibrium condition to return to it. This time is essential for the equalization of intrinsic processes of the machine components, which defines a new ideal pace of work and the advent of adaptation.

Interestingly, within the known universe, natural eternity presents criteria that are impossible to achieve, even for a single simple cell, such as a protist cell, since all beings depend on the environment to live, but it remains constantly changing. This implies an almost continuous process searching of adaptation, which takes the organism away from its ordinary steady state.

It is a paradox that, in order to survive, animals reduce their life span by aging. When we say this, we are not forgetting to consider the genetic characteristics of each species, since the average life expectancy of an animal is intrinsic to its species. However, it is also important to remember that the characteristics of each species result from several mutations over time, which generates adaptive mechanisms that have increased their ability to deal with environmental stressors.

The loss of function of a species within its ecosystem or cells within an organ may be showing that the limits of the stress principle have been "transgressed". That is, the intensity and/or the duration of the stress exceeded the capacity of the organism live to process information extracted from the stressor and reprogram its physiological mechanisms, activating its adaptability process, while its internal balance is preserved.

Understanding their origin, it may be easier to visualize the stress in its many forms and learn, in fact, to deal with it. 


\section{Conflicts of Interest}

The authors declare no conflicts of interest regarding the publication of this paper.

\section{References}

[1] Chao, E.Y.S., Armiger, R.S., Yoshida, H., Lim, J. and Haraguchi, N. (2007) Virtual Interactive Musculoskeletal System (VIMS) in Orthopaedic Research, Education and Clinical Patient Care. Journal of Orthopaedic Surgery Research, 2, 2-21. https://doi.org/10.1186/1749-799X-2-2

[2] Levac, D.E., Huber, M.E. and Sternad, D. (2019) Learning and Transfer of Complex Motor Skills in Virtual Reality: A Perspective Review. Journal of NeuroEngineering and Rehabilitation, 16, 121. https://doi.org/10.1186/s12984-019-0587-8

[3] Borges, V.H., Stelling de Castro, M.C., de Moura, C.A. and Cortez, C.M. (2019) Model for the Storage Control in an Endocrine Gland Based on Lyapunov Function. AIP Conference Proceedings, 2186, Article ID: 130006. https://doi.org/10.1063/1.5138055

[4] Almeida, J., Thadeu, F.C., Silva, D. and Cortez, C.M. (2019) Estimating the Position of Sites for Ligands in Serum Albumins from Spectrofluorimetric Data. AIP Conference Proceedings, 2186, Article ID: 130005. https://doi.org/10.1063/1.5138054

[5] Andalibi, V., Hokkanen, H. and Vanni, S. (2019) Controlling Complexity of Cerebral Cortex Simulations-I: CxSystem, a Flexible Cortical Simulation Framework. Neural Computation, 31, 1048-1065. https://doi.org/10.1162/neco_a_01120

[6] Roy, K., Jaiswal, A. and Panda, P. (2019) Towards Spike-Based Machine Intelligence with Neuromorphic Computing. Nature, 575, 607-617. https://doi.org/10.1038/s41586-019-1677-2

[7] Rodrigues, V., Kalil, C., Stelling de Castro, M.C., Silva, D. and Cortez, C.M. (2018) Mathematical-Computational. Modeling in Behavior's Study of Repetitive Discharge Neuronal Circuits. In: da Silva, F.A.B., Carels, N. and Paes Silva Junior, F., Eds., Theoretical and Applied Aspects of Systems Biology, Springer, New York, 243-259.

[8] Navlakha, S., Bar-Joseph, Z. and Barth, A.L. (2018) Network Design and the Brain. Trends of Cognitive Science, 22, 64-78. https://doi.org/10.1016/j.tics.2017.09.012

[9] Dalcin, B.L.G., Cruz, F.A.O, Cortez, C.M. and Passos, E.L. (2015) Applying Backpropagation Neural Network in the Control of Medullary Reflex Pattern. Conference Proceedings, 1702, Article ID: 130006. https://doi.org/10.1063/1.4938913

[10] Pinto, F.C.M., Silva, D., Silva, P.C., Pereira, L.M., Morone, A.R., Costa, W.S., Cortez, C.M. and Sampaio, F.J.B. (2015) Deleterious Effects of Prepubertal Corticosterone Treatment on Rat Prostate. Acta Cirurgica Brasileira, 30, 382-387. https://doi.org/10.1590/S0102-865020150060000002

[11] Pinto, T.M., Wedeman, R.S. and Cortez, C.M. (2011) Computational Modeling of the Electric Potential in Biological Membrane. A Comparison Between Healthy and Cancerous Neurons. BMC Neuroscience (Online), 12, 47-48. https://doi.org/10.1186/1471-2202-12-S1-P47

[12] Pinto, T.M., Wedemann, R.S. and Cortez, C.M. (2011) Modeling the Electric Potential across Neuronal Membranes: The Effect of Fixed Charges on Spinal Ganglion Neurons and Neuroblatoma Cells. PLoS ONE, 9, e96194. https://doi.org/10.1371/journal.pone.0096194

[13] Cardoso, F.R., Cruz, F.A.O., Silva, D. and Cortez, C.M. (2009) Computational Mod- 
eling of Synchronization Process of the Circadian Timing System of Mammals. Biological Cybernetics, 100, 385-393. https://doi.org/10.1007/s00422-009-0309-6

[14] Cruz, F.A.O., Silva, D. and Cortez, C.M. (2008) Simulation of a Spinal Reflex Circuit Model Controlled by a Central Pattern Generator. Far East Journal Applied Mathematics, 33, 307-336.

[15] Sjöström, P.J., Rancz, E.A., Roth, A. and Häusser, M. (2008) Dendritic Excitability and Synaptic Plasticity. Physiological Reviews, 88, 769-840. https://doi.org/10.1152/physrev.00016.2007

[16] Cruz, F.A.O. and Cortez, C.M. (2005) Computer Simulation of a Central Pattern Generator via Kuramoto Model. Physica A: Statist. Theoretical Physics, 353, 258-270. https://doi.org/10.1016/j.physa.2004.12.046

[17] Momma, D., Funakoshi, T., Endo, K., Yokota, M., Fujisaki, K. and Iwasaki, N. (2018) Alteration in Stress Distribution Patterns through the Elbow Joint in Professional and College Baseball Pitchers: Using Computed Tomography Osteoabsorptiometri. Journal of Orthopaetic Science, 23, 948-952. https://doi.org/10.1016/j.jos.2018.06.006

[18] Márquez-Florez, K., Vergara-Amador, E., de Las Casas, E.B. and Garzón-Alvarado, D.A. (2015) Theoretical Distribution of Load in the Radius and Ulna Carpal Joint. Computater in Biology and Medicine, 60, 100-106. https://doi.org/10.1016/j.compbiomed.2015.02.016

[19] Avari, H., Savory, E. and Rogers, K.A. (2016) An in Vitro Hemodynamic Flow System to Study the Effects of Quantified Shear Stresses on Endothelial Cells. Cardiovascular Engineering and Technology, 7, 44-57. https://doi.org/10.1007/s13239-015-0250-x

[20] Kober, C. Hellmich, C., Stübinger, S., Zeilhofer, H.F. and Sader, R. (2015) Anatomical Simulation of the Biomechanical Behavior of the Human Mandible. International Journal of Computerized Dentistry, 18, 333-342.

[21] June, R., Mejia, K., Barone, J. and Fyhrie, D. (2009) Cartilage Stress-Relaxation Is Affected by Both the Charge Concentration and Valence of Solution Cations. Osteoarthritis and Cartilage, 17, 669-676. https://doi.org/10.1016/j.joca.2008.09.011

[22] Anderson, D.D., Goldsworthy, J.K., Shivanna, K., Grosland, N.M., Pedersen, D.R., Thomas, T.P., Tochigi, Y., Marsh, J.L. and Brown, T.D. (2006) Intra-Articular Contact Stress Distributions at the Ankle throughout Stance Phase-Patient-Specific Finite Element Analysis as a Metric of Degeneration Propensity. Biomechanical and Model in Mechanobiology, 5, 82-89. https://doi.org/10.1007/s10237-006-0025-2

[23] Grootaert, M.O.J., Moulis, M., Roth, L., Martinet, W., Vindis, C., Bennett, M.R. and De Meyer, G.R.Y. (2018) Vascular Smooth Muscle Cell Death, Autophagy and Senescence in Atherosclerosis. Cardiovasculae Research, 114, 622-634.

https://doi.org/10.1093/cvr/cvy007

[24] Nakahara, T., Dweck, M.R., Narula, N., Pisapia, D., Narula, J. and Strauss H.W. (2017) Coronary Artery Calcification: From Mechanism to Molecular Imaging. JACC Cardiovascular Imaging, 10, 582-593. https://doi.org/10.1016/j.jcmg.2017.03.005

[25] Gessaghi, V.C., Raschi, M.A., Larreteguy, A.E. and Perazzo, C.A. (2005) Simulación del Efecto del LDL en el Crecimiento de una Placa Aterosclerosa. Mecánica Computacional, 24, 759-771.

[26] Gessaghi, V.C., Raschi, M.A., Larreteguy, A.E. and Perazzo, C.A. (2006) Influencia de las Características Reológicas y no Estacionarias del Flujo Sanguíneo en un Modelo de Crecimiento de Placas Ateroscleróticas. Mecánica Computacional, 25, 759-770. 
[27] Gessaghi, V.C., Larreteguy, A.E., Raschi, M. and Perazzo, C.A. (2007) Influence of Arterial Geometry on a Model for Growth Rate of Atheromas. Journal of Physics Conference Series, 90, Article ID: 012046. https://doi.org/10.1088/1742-6596/90/1/012046

[28] Kenny, A., Plank, M.J. and David, T. (2018) The Role of Astrocytic Calcium and TRPV4 Channels in Neurovascular Coupling. Journal Computational Neuroscience, 44, 97-114. https://doi.org/10.1007/s10827-017-0671-7

[29] Dormanns, K., Brown, R.G. and David, T. (2016) The Role of Nitric Oxide in Neurovascular Coupling. Journal of Theoretical Biology, 394, 1-17. https://doi.org/10.1016/j.jtbi.2016.01.009

[30] Gupta, M., Sarangi, B.R., Deschamps, J., Nematbakhsh, Y., Callan-Jones, A., Margadant, F., Mège, R.M., Lim, C.T., Voituriez, R. and Ladoux, B. (2015) Adaptive Rheology and Ordering of Cell Cytoskeleton Govern Matrix Rigidity Sensing. Nature Communications, 6, 7525. https://doi.org/10.1038/ncomms8525

[31] Fallqvist, B., Kulachenko, A. and Kroon, M. (2014) Modelling of Cross-Linked Actin Networks-Influence of Geometrical Parameters and Cross-Link Compliance. Journal of Theoretical Biology, 350, 57-69. https://doi.org/10.1016/j.jtbi.2014.01.032

[32] Mogilner, A. and Oster, G. (2003) Force Generation by Actin Polymerization II: The Elastic Ratchet and Tethered Filaments. Biophysical Journal, 84, 1591-1605. https://doi.org/10.1016/S0006-3495(03)74969-8

[33] Nejad, A.A., Talebi Z., Cheraghali, D., Shahbani-Zahiri, A. and Norouzi, M. (2018) Pulsatile Flow of Non-Newtonian Blood Fluid inside Stenosed Arteries: Investigating the Effects of Viscoelastic and Elastic Walls, Arteriosclerosis, and Polycythemia Diseases. Computational Methods and Programs in Biomedicine, 154, 109-122. https://doi.org/10.1016/j.cmpb.2017.11.016

[34] Prado, C.M., Ramos, S.G., Alves-Filho, J.C., Elias Jr., J., Cunha, F.Q. and Rossi, M.A. (2006) Turbulent Flow/Low Wall Shear Stress and Stretch Differentially Affect Aorta Remodeling in Rats. Journal of Hypertension, 24, 503-515. https://doi.org/10.1097/01.hjh.0000209987.51606.23

[35] Silva, J.R., Vivanco-Carlevari, A., Barrientos, M., Martínez, C., Salazar, L.A. and Krause, M. (2017) Biological Stress Reactivity as an Index of the Two Polarities of the Experience Model. Psychoneuroendocrinology, 84, 83-86. https://doi.org/10.1016/j.psyneuen.2017.06.016

[36] Rom, O. and Reznick, A.Z. (2016) The Stress Reaction: A Historical Perspective. Advanced in Experimental Medicine Biology, 905, 1-4. https://doi.org/10.1007/5584_2015_195

[37] Sevillano-Gracia, M.D., Manso-Calderón, R. and Cacabelos-Pérez, P. (2007) Comorbidity in the Migraine: Depression, Anxiety, Stress and Insomnia. Reviews in Neurology, 45, 400-405. https://doi.org/10.33588/rn.4507.2007122

[38] Cortez, C.M. (1991) Stress and Its Physiological Inferences. A Folha Médica, 103, 175-181.

[39] Cortez, C.M., Cruz, F.A.O. and Silva, D. (2008) Psychicological and Physiological Responses to Stress: A Review Based on Results from PET and MRI Studies. Brazilian Archives of Biology and Technology, 51, 7-12. https://doi.org/10.1590/S1516-89132008000700003

[40] Irgens, F. (2008) Continuum Mechanics. Springer, New York.

[41] Teodor, M. and Atanackovic, A.G. (2000) Theory of Elasticity for Scientists and Engineers. Springer, New York. https://doi.org/10.1007/978-1-4612-1330-7 
[42] Cima, I., Corazza, N., Dick, B., Fuhrer, A., Herren, S., Jakob, S., Ayuni, E., Mueller, C. and Brunner, T. (2004) Intestinal Epithelial Cells Synthesize Glucocorticoids and Regulate T Cell Activation. Journal of Experimental Medicine, 200, 1635-1646. https://doi.org/10.1084/jem.20031958

[43] Samad, N. and Halleem, D.J. (2007) Serotonin-1A Receptor Responsiveness in Stress and Following Adaptation to Stress. Pakistan Journal Pharmaceutical Sciences, 20, 115-119.

[44] Leclerc, C., Lesage, A. and Ricard, N. (1997) Relevance of the Stress, Coping Paradigm in the Elaboration of a Stress Management Model for Schizophrenics. Sante Mentale au Quebec, 22, 233-256. https://doi.org/10.7202/032424ar

[45] Selye, H. (1946) The General Adaptation Syndrome and the Diseases of Adaptation. Journal Clinical Endocrinology and Metabolism, 6, 117-196. https://doi.org/10.1210/jcem-6-2-117

[46] Lubliner, J. (2006) Plasticity Theory. Pearson Education, Inc., London.

[47] Hegyi, G., Vincze, G. and Szasz, A. (2012) On the Dynamic Equilibrium in Homeostasis. Open Journal of Biophysics, 2, Article ID: 21417.

[48] Rhoades, R.A. and Tanner, G.A. (2003) Medical Physiology. 2th Edition, Lippincott, Williams \& Williams, Inc., Philadelphia.

[49] Jackson, M.C. (2000) System Approaches to Management. Kluwer Academic Pub., New York.

[50] Cannon, W.B. (1914) The Emergency Function of the Adrenal Medulla in Pain and the Major Emotions. American Journal of Physiology, 33, 356-372. https://doi.org/10.1152/ajplegacy.1914.33.2.356

[51] Rabasa, C. and Dickson, S.L. (2016) Impact of Stress on Metabolism and Energy Balance. Current Opinion Behavioral Science, 9, 71-77. https://doi.org/10.1016/j.cobeha.2016.01.011

[52] Alemany, M. (2012) Do the Interactions between Glucocorticoids and Sex Hormones Regulate. The Development of the Metabolic Syndrome? Front Endocrinology (Lausanne), 3, 27. https://doi.org/10.3389/fendo.2012.00027

[53] Meyer, J.S. (1985) Biochemical Effects of Corticosteroids on Neural Tissues. Physiology Reviews, 65, 946-1020. https://doi.org/10.1152/physrev.1985.65.4.946

[54] Souza, D.B., Silva, D., Cortez, C.M., Costa, W.S. and Sampaio, J.B.S. (2015) Functional Roles of Corticosterone and Stress in Penile Morphology. In: Santulli, G., Ed., Adrenal Glands. From Pathophysiology to Clinical Evidence, Nova Science Publishers, New York, Chapter 14, 299-306.

[55] Ribeiro, G.S., Souza, D.B., Cortez, C.M., Silva, D., Costa, W.S. and Sampaio, F.J.B. (2014) Effects of Prepubertal Corticosterone Treatment on Urinary Bladder. Acta Cirurgica Brasileira, 29, 55-59. https://doi.org/10.1590/S0102-86502014001700011

[56] Ribeiro, G., Cardoso, L.E.M., Souza, D.B., Cortez, C.M., Silva, D., Costa, W.S. and Sampaio, F.J.B. (2012) Prepubertal Corticosterone Treatment Affects Bladder Wall in Rats. Theoretical Journal of Urology, 187, 244. https://doi.org/10.1016/j.juro.2012.02.676

[57] Seckl, J.R. (2004) Prenatal Glucocorticoids and Longterm Programming. European Journal of Endocrinology, 151, U49-U62. https://doi.org/10.1530/eje.0.151u049

[58] Cortez, C.M., Costa, W.S., Babinski, M.A. and Chagas, M.A. (2003) Morphometrical and Stereological Analysis of the Superior Cervical Glanglion of Rattus norvegicus Submitted to Chronic treatment with Cortisol. International Journal of Morphology, 21, 211-226. https://doi.org/10.4067/S0717-95022003000300006 
[59] Rosmond, R. and Björntorp, P. (2000) The Hypothalamic-Pituitary-Adrenal Axis Activity as a Predictor of Cardiovascular Disease, Type 2 Diabetes and Stroke. Journal International Medicine, 247, 188-197. https://doi.org/10.1046/j.1365-2796.2000.00603.x

[60] Kuo, T., McQueen, A., Chen, T.C. and Wang, J.C. (2015) Regulation of Glucose Homeostasis by Glucocorticoids. Advances Experimental Medicine and Biology, 872, 99-126. https://doi.org/10.1007/978-1-4939-2895-8_5

[61] Alan, S.I. and Alan, B. (2017) Side Effects of Glucocorticoids. In: Malangu, N., Ed., Pharmakinetics and Adverse Effects of Drugs. Mechanisms and Risks Factors, IntechOpen, London. https://doi.org/10.5772/intechopen.72019

[62] Braun, T.P. and Marks, D.L. (2015) The Regulation of Muscle Mass by Endogenous Glucocorticoids. Frontier in Physiology, 6, Art. 12.

https://doi.org/10.3389/fphys.2015.00012

[63] Patterson-Buckendahl, P., Rusnák, M., Fukuhara, K. and Kvetnanšý, R. (2001) Repeated Immobilization Stress Reduces Rat Vertebral Bone Growth and Osteocalcin. American Journal of Regulatory and Integrative Physiology, 280, R79-R86. https://doi.org/10.1152/ajpregu.2001.280.1.R79

[64] Madalena, K.M. and Lerch, J.K. (2017) The Effect of Glucocorticoid and Glucocorticoid Receptor Interactions on Brain, Spinal Cord, and Glial Cell Plasticity. Neura Plasticity, 2017, Article ID: 8640970. https://doi.org/10.1155/2017/8640970

[65] Chatham, W.W. (2019) Glucocorticoid Effects on the Immune System. UptoDate. https://www.uptodate.com/contents/glucocorticoid-effects-on-the-immune-system

[66] Chen, Y., Zhang, M. and Zheng, Y. (2018) Glucocorticoids Inhibit Production of Exosomes Containing Inflammatory MicrorNA-155 in Lipopolysaccharide-Induced Macrophage Inflammatory Responses. International Journal of Clinical and Experimental Pathology, 11, 3391-3397. http://www.ijcep.com

[67] Bilandzić, N., Zurić, M., Lojkić, M., Simić, B., Milić, D. and Barac, I. (2006) Cortisol and Immune Measures in Boars Exposed to Three-Day Administration of Exogenous Adrenocorticotropic Hormone. Veterinary Research Communications, 30, 433-444. https://doi.org/10.1007/s11259-006-3315-3

[68] Romundstad, L., Breivik, H. and Stubhaug, A. (2005) Glucocorticoids Reduce Acute Postoperative Pain. Tidsskr Nor Laegeforen, 125, 2507-2508.

[69] Liberman, A.C., Budziñski, M.L., Sokn, C., Gobbini, R.P., Steininger, A. and Arzt, E. (2018) Regulatory and Mechanistic Actions of Glucocorticoids on T and Inflammatory Cells. Frontier in Endocrinology, 9, 235. https://doi.org/10.3389/fendo.2018.00235

[70] Reiche, E.M., Morimoto, H.K. and Nunes, S.M. (2005) Stress and Depression-Induced Immune Dysfunction: Implications for the Development and Progression of Cancer. International Reviews of Psychiatry, 17, 515-527. https://doi.org/10.1080/02646830500382102

[71] McKay, L.I., John, A. and Cidlowski, J.A. (2003) Physiologic and Pharmacologic Effects of Corticosteroids. In: Kufe, D.W., et al., Eds., Holland-Frei Cancer Medicine, 6th Edition, BC Decker, Hamilton. https://www.ncbi.nlm.nih.gov/books/NBK13780/

[72] Leserman, J., Petitto, J.M., Gu, H., Gaynes, B.N., Barroso, J., Golden, R.N., Perkins, D.O., Folds, J.D. and Evans, D.L. (2002) Progression to AIDS, a Clinical AIDS Condition and Mortality: Psychosocial and Physiological Predictors. Psychology Medicine, 32, 1059-1073. https://doi.org/10.1017/S0033291702005949

[73] Souza, D., Silva D., Silva, C.M.C., Sampaio, F.J.B., Costa, W.S. and Cortez, C.M.. 
(2011) Effects of Immobilization Stress on Kidney of Male Rats Wistar. A Morphometrical and Stereological Analysis. Kidney and Blood Pressure Research, 343, 424-429. https://doi.org/10.1159/000328331

[74] Souza, D.B., Silva, D., Cortez, C.M., Sampaio, J.B.S. and Costa, W.S. (2011) Effects of Chronic Stress on Penile. Journal of Andrology, 33, 735-741.

https://doi.org/10.2164/jandrol.111.014225

[75] Pervanidou, P. and Chrousos, G.P. (2010) Neuroendocrinology of Posttraumatic Stress Disorder. Program in Brain Research, 182, 149-160.

https://doi.org/10.1016/S0079-6123(10)82005-9

[76] O’Connor, D.B., Hendrickx, H. and Dadd, T. (2009) Cortisol Awakening Rise in Middle-Aged Women in Relation to Psychological Stress. Psychoneuroendocrinology, 34, 1486-1494. https://doi.org/10.1016/j.psyneuen.2009.05.002

[77] Lang, J., Ochsmann, E., Kraus, T. and Lang, J.W. (2012) Psychosocial Work Stressors as Antecedents of Musculoskeletal Problems: A Systematic Review and Meta-Analysis of Stability-Adjusted Longitudinal Studies. Social Science \& Medicine, 75, 1163-1174. https://doi.org/10.1016/j.socscimed.2012.04.015

[78] Eller, N.H., Kristiansen, J. and Hansen, A.M. (2011) Long-Term Effects of Psychosocial Factors of Home and Work on Biomarkers of Stress. International Journal Psychophysiology, 79, 195-202. https://doi.org/10.1016/j.ijpsycho.2010.10.009

[79] Katan, M. and Christ-Crain, M. (2010) The Stress Hormone Copeptin: A New Prognostic Biomarker in Acute Illness. Swiss Medicine Weekly, 140, w13101. https://doi.org/10.4414/smw.2010.13101

[80] Nater, U.M., La, Marca, R., Florin, L. and Moses, A. (2006) Stress-Induced Changes in Human Salivary Alpha-Amylase Activity-Associations with Adrenergic Activity. Psychoneuroendocrinology, 31, 49-58. https://doi.org/10.1016/j.psyneuen.2005.05.010

[81] Boileau, K., Barbeau, K., Sharma, R. and Bielajew, C. (2019) Ethnic Differences in Diurnal Cortisol Profiles in Healthy Adults: A Meta-Analysis. Brazilian Journal of Health Psychology, 24, 806-827. https://doi.org/10.1111/bjhp.12380

[82] Urizar, G.G., Yim, I.S., Kofman, Y.B., Tilka, N., Miller, K., Freche, R. and Johnson, A. (2019) Ethnic Differences in Stress-Induced Cortisol Responses: Increased Risk for Depression during Pregnancy. Biological Psychology, 147, Article ID: 107630. https://doi.org/10.1016/j.biopsycho.2018.12.005

[83] Deer, L.K., Shields, G.S., Ivory, S.L., Hostinar, C.E. and Telzer, E.H. (2018) Racial/Ethnic Disparities in Cortisol Diurnal Patterns and Affect in Adolescence. Development and Psychopathology, 30, 1977-1993. https://doi.org/10.1017/S0954579418001098

[84] Lloyd, S. (2008) Quantum Information Matters. Science, 319, 1209-1211. https://doi.org/10.1126/science.1154732

[85] Karamouz, M., Nokhandan, A., Kerachian, R. and Maksimovic, C. (2013) Design of Online River Water Quality Monitoring Systems Using the Entropy Theory: A Case Study. Environmental Monitoring and Assessment, 155, 63-81. https://doi.org/10.1007/s10661-008-0418-z

[86] Lishmanov, I.B., Lasukova, T.V., Afanasev, S.A., Maslov, L.N., Krotenko, N.M. and Naryzhnaia, N.V. (1997) Effect of Cold Stress on the Contractile Activity, Carbohydrate and Energy Metabolism in the Isolated Rat Heart. Patologicheskaia Fiziologiia i Eksperimentalnaia Terapiia, 1, 28-31.

[87] Huang, C.J., Stewart, J.K., Franco, R.L. Evans, R.K., Lee, Z.P., Cruz, T.D., Webb, H.E. and Acevedo, E.O. (2011) LPS-Stimulated Tumor Necrosis Factor-Alpha and 
Interleukin-6 Mrna and Cytokine Responses Following Acute Psychological Stress. Psychoneuroendocrinology, 36, 1553-1561.

https://doi.org/10.1016/j.psyneuen.2011.04.009

[88] Owen, N. and Steptoe, A. (2003) Natural Killer Cell and Proinflammatory Cytokine Responses to Mental Stress: Associations with Heart Rate and Heart Rate Variability. Biological Psychology, 63, 101-115. https://doi.org/10.1016/S0301-0511(03)00023-1

[89] Şimşek, T., Şimşek, H.U. and Cantürk, N.Z. (2014) Response to Trauma and Metabolic Changes: Posttraumatic Metabolism. Ulusal Cerrahi Dergisi, 30, 153-159. https://doi.org/10.5152/UCD.2014.2653

[90] Lovell, M.A. and Markesbery, W.R. (2007) Oxidative Damage in Mild Cognitive Impairment and Early Alzheimer's Disease. Journal of Neuroscience Research, 85, 3036-3040. https://doi.org/10.1002/jnr.21346

[91] Neha, K., Haider, M.R., Pathak, A. and Yar, M.S. (2019) Medicinal Prospects of Antioxidants: A Review. European Journal of Medical Chemistry, 178, 687-704. https://doi.org/10.1016/j.ejmech.2019.06.010

[92] Raza, C., Anjum, R. and Shakeel, N.U.A. (2019) Parkinson's Disease: Mechanisms, Translational Models And Management Strategies. Life Science, 226, 77-90. https://doi.org/10.1016/j.lfs.2019.03.057

[93] Klaunig, J.E. (2018) Oxidative Stress and Cancer. Current Pharmacological Design, 24, 4771-4778. https://doi.org/10.2174/1381612825666190215121712

[94] Cobley, J.N., Fiorello, M.L. and Bailey, D.M. (2018) 13 Reasons Why the Brain Is Susceptible to Oxidative Stress. Redox Biology, 15, 490-503.

https://doi.org/10.1016/j.redox.2018.01.008

[95] Guajardo, M.H., Terrasa, A.M. and Catalã, A. (2006) Lipid-Protein Modifications during Ascorbate- $\mathrm{Fe}^{2+}$ Peroxidation of Photoreceptor Membranes: Protective Effect of Melatonin. Journal of Pineal Research, 41, 201-210. https://doi.org/10.1111/j.1600-079X.2006.00352.x

[96] Epel, E.S., Blackburn, E.H., Lin, J., Dhabhar, F.S., Adler, N.E., Morrow, J.D. and Cawthon, R.M. (2004) Accelerated Telomere Shortening in Response to Life Stress. Proceeding of National Academy of Sciences USA, 101, 17312-17315. https://doi.org/10.1073/pnas.0407162101

[97] Arsenis, N.C., You, T., Ogawa, E.F., Tinsley, G.M. and Zuo, L.P. (2017) Physical Activity and Telomere Length: Impact of Aging and Potential Mechanisms of Action. Oncotarget, 8, 45008-45019. https://doi.org/10.18632/oncotarget.16726

[98] Zhao, Y., Hoshiyama, H., Shay, J.W. and Wright, W.E. (2008) Quantitative Telomeric Overhang Determination Using a Double-Strand Specific Nuclease. Nucleic Acids Research, 36, 14-19. https://doi.org/10.1093/nar/gkm1063

[99] Epel, E.S, Lin, J., Wilhlm, F.H., Wilkowitz, O.M., Cawthon, R., Adler, N.E., Dolbier, C., Mendes, W.B. and Blackburn, E.H. (2006) Cell Aging in Relation to Stress Arousal and Cardiovascular Disease Risk Factors. Psychoneuroendocrinology, 31, 277-287. https://doi.org/10.1016/j.psyneuen.2005.08.011

[100] Kiecolt-Glaser, J.K. and Glaser, R. (2010) Psychological Stress, Telomeres, and Telomerase. Brain, Behavior and Immunity, 24, 529-530. https://doi.org/10.1016/j.bbi.2010.02.002

[101] Kiecolt-Glaser, J.K., Preacher, K.J., MacCallum, R.C., Atkinson, C., Malarkey, W.B. and Glaser, R. (2003) Chronic Stress and Age-Related Increases in the Proinflammatory Cytokine IL-6. Proceedings of National Academy of Sciences USA, 100, 9090-9095. https://doi.org/10.1073/pnas.1531903100 
[102] Crous-Bou, M., Molinuevo, J.L. and Sala-Vila, A. (2019) Plant-Rich Dietary Patterns, Plant Foods and Nutrients, and Telomere Length. Advanced Nutrition, 10, S296-S303. https://doi.org/10.1093/advances/nmz026

[103] Aviv, A. (2004) Telomeres and Human Aging: Facts and Fibs. Science of Aging Knowledge Environment, 51, 43. https://doi.org/10.1126/sageke.2004.51.pe43

[104] Green, P.D., Sharma, N.K. and Santos, J.H. (2019) Telomerase Impinges on the Cellular Response to Oxidative Stress through Mitochondrial ROS-Mediated Regulation of Autophagy. International Journal of Molecular Sciences, 20, 1509. https://doi.org/10.3390/ijms20061509

[105] Kosebent, E.G., Uysal, F. and Ozturk, S. (2018) Telomere Length and Telomerase Activity during Folliculogenesis in Mammals. Journal Reproduction and Development, 64, 477-484. https://doi.org/10.1262/jrd.2018-076

[106] Ahmed, W. and Lingner, J. (2018) Impact of Oxidative Stress on Telomere Biology. Differentiation, 99, 21-27. https://doi.org/10.1016/j.diff.2017.12.002

[107] Zannas, A.S. (2016) Editorial Perspective: Psychological Stress and Epigenetic Aging-What Can We Learn and How Can We Prevent? Journal of Child Psychology and Psychiatry, 57, 674-675. https://doi.org/10.1111/jcpp.12535

[108] Hamilton, K.L. and Miller, B.F. (2016) What Is the Evidence for Stress Resistance and Slowed Aging? Experimental Gerontology, 82, 67-72.

https://doi.org/10.1016/j.exger.2016.06.001

[109] Vitlic, A., Lord, J.M. and Phillips, A.C. (2014) Stress, Ageing and Their Influence on Functional, Cellular and Molecular Aspects of the Immune System. Age (Dordr), 36, 9631. https://doi.org/10.1007/s11357-014-9631-6

[110] Calcinotto, A., Kohli, J., Zagato, E., Pellegrini, L., Demaria, M. and Alimonti, A. (2019) Cellular Senescence: Aging, Cancer and Injury. Physiology Reviews, 99, 1047-1078. https://doi.org/10.1152/physrev.00020.2018

[111] de Magalhães, J.P. and Passos, J.F. (2018) Stress, Cell Senescence and Organismal Ageing. Mechanisms of Ageing and Development, 170, 2-9. https://doi.org/10.1016/j.mad.2017.07.001

[112] Miller, G.E., Chen, E. and Parker, K.J. (2011) Psychological Stress in Childhood and Susceptibility to the Chronic Diseases of Aging: Moving Toward a Model of Behavioral and Biological Mechanisms. Psychology Bulletin, 137, 959-997. https://doi.org/10.1037/a0024768

[113] Hammes, G.G. (2000) Thermodynamics and Kinetics for the Biological Sciences. John Wiley \& Sons, New York.

[114] Clausius, R. (1850) Über die Bewegende Kraft der Wärme und Die Gesetze, Welche Sich Daraus für die Wärmelehre Selbst Ableiten Lassen. Annalen der Physik, 155, 368-397. https://doi.org/10.1002/andp.18501550306

[115] Leonidovich, K.A. (2017) Statistical Mechanics and the Physics of Many-Particle Model Systems. World Scientific Publishing, London.

[116] Gemmer, J., Otte, A. and Mahler, G. (2001) Quantum Approach to a Derivation of the Second Law of Thermodynamics. Physics Review Letters, 86, 1927-1930. https://doi.org/10.1103/PhysRevLett.86.1927

[117] Grandy, W.T. (1987) Foundations of Statistical Mechanics, Vols 1 and 2. Reidel, Dordrecht. https://doi.org/10.1007/978-94-009-3867-0_1

[118] Chakrabarti, C.G. and Chakrabarty, I. (2007) Boltzmann Entropy: Probability and Information. Romanian Journal of Physics, 52, 559-564.

[119] Linde, A. (2007) Sinks in the Landscape, Boltzmann Brains, and the Cosmological 
Constant Problem. Jounal of Cosmology and Astroparticles Physics, 1, 22. https://doi.org/10.1088/1475-7516/2007/01/022

[120] Swenson, R. (2000) Spontaneous Order, Autocatakinetic Closure, and the Development of Space-Time. Annals of N.Y. Academy of Sciences, 901, 311-319. https://doi.org/10.1111/j.1749-6632.2000.tb06290.x

[121] Swenson, R. and Turvey, M.T. (1991) Thermodynamic Reasons for Perception-Action Cycles. Ecological Psychology, 3, 317-348. https://doi.org/10.1207/s15326969eco0304_2

[122] Albrecht, A. and Sorbo, L. (2004) Can the Universe Afford Inflation? Physics Reviews $D$, 70, Article ID: 63528. https://doi.org/10.1103/PhysRevD.70.063528

[123] Vijver, G., Salthe, S.N. and Delpos, M. (1998) Evolutionary Systems: Biological and Epistemological Perspectives on Selection Self-Organization. Springer Science, Business Media, BV, Berlin.

[124] Prigogine, I., Geheniau, J., Gunzig, E. and Nardone, P. (1988) Thermodynamics of Cosmological Matter Creation. Proceeding National of Academy Sciences, USA, 85, 7428-7432. https://doi.org/10.1073/pnas.85.20.7428

[125] Lucia, U. and Grazzine, G. (2015) The Second Law Today: Using Maximum-Minimum Entropy Generation. Entropy, 17, 7786-7797. https://doi.org/10.3390/e17117786

[126] Nicolis, G. and Prigogine, I. (1977) Self Organization in Non-Equilibrium Systems. J Wiley \& Sons, New York, Chaps. III and IV.

[127] Lowe, J.P. (1988) Entropy: Conceptual Disorder. Journal of Chemistry Education, 65, 403-406. https://doi.org/10.1021/ed065p403

[128] Leff, H.S. (1996) Thermodynamic Entropy: The Spreading and Sharing of Energy. American Journal of Physics, 64, 1261-1271. https://doi.org/10.1119/1.18389

[129] Jaynes, E.T. (1957) Information Theory and Statistical Mechanics. Physics Reviews, 106, 620-630. https://doi.org/10.1103/PhysRev.106.620

[130] Shannon, C.E. (1951) Prediction and Entropy. The Bell System Technical Journal, 30, 50-64. https://doi.org/10.1002/j.1538-7305.1951.tb01366.x

[131] Brillouin, L. (2004) Science and Information Theory. Dover, Mineola.

[132] Callen, H.B. (1985) Thermodynamics and an Introduction to Thermostatics. 2nd Edition, John Wiley \& Sons Inc., Singapora.

[133] Zhou, X.Y., Rong, C., Lu, T., Zhou, P.P. and Liu, S. (2016) Information Functional Theory: Electronic Properties as Functionals of Information for Atoms and Molecules. Journal of Physics Chemistry A, 120, 3634-3642. https://doi.org/10.1021/acs.jpca.6b01197

[134] Krause, P. (2019) Information Theory and Medical Decision Making. Studies in Health Technology Informatics, 263, 23-34.

[135] Nayyar, S., Downar, E., Beheshti, M., Liang, T., Massé, S., Magtibay, K., Bhaskaran, A., Saeed, Y., Vigmond, E. and Nanthakumar, K. (2019) Information Theory to Tachycardia Therapy: Electrogram Entropy Predicts Diastolic Microstructure of Reentrant Ventricular Tachycardia. American Journal of Physiological-Heart and Circulatory Physiology, 316, H134-H144. https://doi.org/10.1152/ajpheart.00581.2018

[136] Ferrario, P.G. and König, I.R. (2018) Transferring Entropy to the Realm of GxG Interactions. Brief Bioinformatics, 19, 136-147.

[137] Castellano, G., González-Santander, J.L., Lara, A. and Torrens, F. (2013) Classification of Flavonoid Compounds by Using Entropy of Information Theory. Phytochemistry, 93, 182-191. https://doi.org/10.1016/j.phytochem.2013.03.024 
[138] Castellano, G. and Torrens, F. (2015) Information Entropy-Based Classification of Triterpenoids and Steroids from Ganoderma. Phytochemistry, 116, 305-313. https://doi.org/10.1016/j.phytochem.2015.05.008

[139] Riggs, J.E. (1993) Aging and Mortality: Manifestations of Increasing Informational Entropy of the Genome? Mechanical of Ageing and Development, 66, 249-256. https://doi.org/10.1016/0047-6374(93)90012-G

[140] Mak, C.H., Pham, P. and Goodman, M.F. (2019) Random Walk Enzymes: Information Theory, Quantum Isomorphism, and Entropy Dispersion. Journal Physics Chemistry A, 123, 3030-3037. https://doi.org/10.1021/acs.jpca.9b00910

[141] Watanabe, N. (2016) Note on Entropies for Quantum Dynamical Systems. Philosophical Transactions of the Royal Society $A, 28,374$. https://doi.org/10.1098/rsta.2015.0240

[142] Gatlin, L.L. (1974) Conservation of Shannon's Redundancy for Proteins. Journal of Molecular Evolution, 3, 189-208. https://doi.org/10.1007/BF01797453

[143] León, A. (1990) Coevolution: New Thermodynamic Theorems. Journal of Theoretical Biology, 147, 205-212. https://doi.org/10.1016/S0022-5193(05)80052-9

[144] Ofria, C., Adami, C. and Collier, T.C. (2003) Selective Pressures on Genomes in Molecular Evolution. Journal of Theoretical Biology, 222, 477-483. https://doi.org/10.1016/S0022-5193(03)00062-6

[145] Lan, G. and Tu, Y. (2016) Information Processing in Bacteria: Memory, Computation, and Statistical Physics: A Key Issues Review. Reports on Program Physics, 79, Article ID: 052601. https://doi.org/10.1088/0034-4885/79/5/052601

[146] Deb, B.M. (1979) On Some “Local” Force Densities and Stress Tensors in Molecular Quantum Mechanics. Journal of Physics B, 12, 3857-3871. https://doi.org/10.1088/0022-3700/12/23/013

[147] Bazant, M.Z. (2013) Theory of Chemical Kinetics and Charge Transfer Based on Nonequilibrium Thermodynamics. Accounts of Chemical Research, 46, 1144-1160. https://doi.org/10.1021/ar300145c

[148] Olberding, J.E., Thouless, M.D., Arruda, E.M. and Garikipati, K. (2010) The Non-Equilibrium Thermodynamics and Kinetics of Focal Adhesion Dynamics. PLoS ONE, 5, e12043. https://doi.org/10.1371/journal.pone.0012043

[149] Davies, K. (1995) Oxidative Stress: The Paradox of Aerobic Life. Biochemical Society Symposium, 61, 1-31. https://doi.org/10.1042/bss0610001

[150] Ma, S.P. and Zahalak, G.I. (1991) A Distribution-Moment Model of Energetics in Skeletal Muscle. Journal of Biomechemistry, 24, 1-35. https://doi.org/10.1016/0021-9290(91)90323-F

[151] Luo, L., Molnar, J., Ding, H., Lv, X. and Spengler, G. (2006) Physicochemical Attack against Solid Tumors Based on the Reversal of Direction of Entropy Flow: An Attempt to Introduce Thermodynamics in Anticancer Therapy. Diagnostic Pathology, 1, 43. https://doi.org/10.1186/1746-1596-1-43 